Background: Patients with rheumatoid arthritis (RA) who have received multiple biologics or targeted therapies over time tend to have more refractory and more severe disease, which may lead to worse clinical response to treatment.

Objectives: We used data from the ACR RISE registry to assess whether disease severity was greater in those who received sarilumab shortly after its FDA approval (May 2017) than in subsequent time periods and to evaluate the effectiveness of sarilumab in populations with various degrees of disease severity. Methods: Patients with RA who initiated sarilumab treatment in the period 20172020 were identified in the ACR RISE registry and divided into Cohort 1 (2017, year of the FDA approval) and the calendar year-based Cohorts 2-4 (2018-2020). Patient demographics, RA-related features, and comorbidities were determined using data prior to sarilumab initiation. The cohorts were compared using chisquare test (categorical variables) and a nonparametric test (continuous variables). Sarilumab effectiveness was assessed using 3 cohorts assembled based on progressively restrictive criteria: Active Disease cohort (Clinical Disease Activity Index [CDAI] $>10$ or Routine Assessment of Patient Index Data 3 [RAPID3] $>6$, and C-reactive protein, if measured, $\geq 8 \mathrm{mg} / \mathrm{L}$ ), TARGET Eligibility cohort (patients who satisfied enrolment criteria for TARGET, ${ }^{1}$ a Phase 3 sarilumab trial in patients with RA and an inadequate response to TNF inhibitors), and TARGET Baseline cohort (patients from TARGET Eligibility cohort with characteristics weighted to match those from the TARGET trial baseline ${ }^{1}$ using the matching-adjusted indirect comparison method $^{2}$ ). In all 3 effectiveness cohorts, mean changes in CDAI and RAPID3 at 6 and 12 months post-initiation of sarilumab were evaluated using a model adjusted for baseline score, age, sex, race, calendar year, and seropositivity.

Results: A total of 2949 patients, treated by 585 rheumatologists, initiated sarilumab treatment in the period 2017-2020. The 4 yearly cohorts were relatively similar in terms of patients' age, sex, race, and most clinical characteristics. However, patients receiving sarilumab shortly after FDA approval (Cohort 1) had more ambulatory visits, a greater number of previously used non-TNFi biologics (particularly tocilizumab), and a higher comorbidity burden, and were more likely to be current users of glucocorticoids or opioids than sarilumab initiators in the subsequent 3 years. In the 3 cohorts used to assess sarilumab effectiveness, the greatest improvement was observed in the TARGET Baseline cohort, which also had the greatest mean baseline CDAI score (43), compared with the other two (24 both).

Conclusion: In this real-world cohort, we observed modest evidence for channeling of patients with greater RA severity and greater prior exposure to nonTNFi biologics to sarilumab shortly after its FDA approval. This cohort effect did not diminish the effectiveness of sarilumab. All cohorts showed improvement, with the greatest clinical improvement observed in the cohort with the highest baseline CDAl score who most closely resembled those enrolled in a phase 3 trial of patients with an inadequate response to TNF inhibitors.

REFERENCES:

[1] Fleischmann R, et al. Arthritis Rheumatol 2017;69:277-290.

[2] Signorovitch JE et al. Value Health 2012;15:940-7.

Adjusteda Improvement in CDAI With Progressive Restriction in Inclusion Criteria

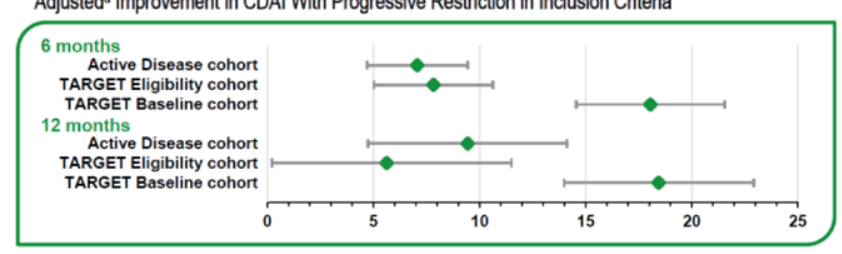

sAdjusted for age, sex, race, calendar year, seropositivity, and baseline $\mathrm{CDA}$

Adjusteda Improvement in RAPID3 With Progressive Restriction in Inclusion Criteria

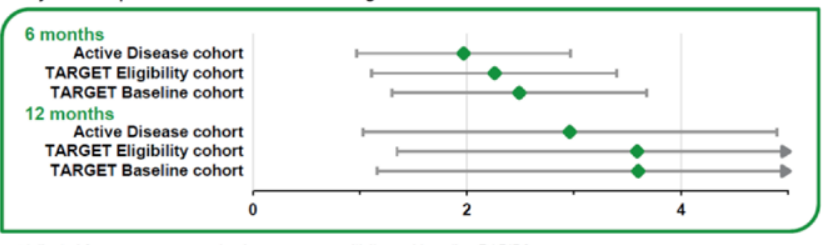

aAdjusted for age, sex, race, calendar year, seropositivity, and baseline RAPID3

Figure 1. Adjusted improvements in CDAI and RAPID3
Acknowledgements: This study was sponsored by Sanofi. Medical writing support was provided by Vojislav Pejović, PhD (Eloquent Medical Affairs, division of Envision Pharma Group) and funded by Sanofi.

Disclosure of Interests: Stefano Fiore Employee of: Sanofi, Lang Chen: None declared, Cassie Clinton Consultant of: Information available in profile, Huifeng Yun Grant/research support from: Research support for Pfizer, Amy Praestgaard Employee of: Sanofi, Kerri Ford Employee of: Sanofi, Jeffrey Curtis Consultant of: Received consulting and research grants from AbbVie, Amgen, BMS, Lilly, Gilead, GSK, Janssen, Myriad, Pfizer, Roche, Samsung, Sandoz, Sanofi, UCB, Grant/research support from: Received consulting and research grants from AbbVie, Amgen, BMS, Lilly, Gilead, GSK, Janssen, Myriad, Pfizer, Roche, Samsung, Sandoz, Sanofi, UCB

DOI: 10.1136/annrheumdis-2021-eular.3655

\section{POS0639 \\ LONG-TERM EFFECTIVENESS OF THE RITUXIMAB HALF DOSE REGIMEN FOLLOWING A RESPONSE TO THE LICENSED DOSE IN RHEUMATOID ARTHRITIS}

L. Garcia-Montoya ${ }^{1,2}$, M. Y. Md Yusof ${ }^{1,2}$, G. Eugénio ${ }^{1,2}$, J. B. Candelier ${ }^{1,2}$ S. Das ${ }^{1,2}$, E. Vital ${ }^{1,2}$, P. Emery ${ }^{1,2} .{ }^{1}$ Leeds Institute of Rheumatic and Musculoskeletal Medicine, University of Leeds, Leeds, United Kingdom; ${ }^{2}$ Leeds Biomedical Research Centre, NIHR, Leeds, United Kingdom

Background: Following initial treatment with rituximab (RTX) full dose (2x1000mg), B-cell numbers are often lower than baseline. The half dose regime (2x500mg) has been reported to be similarly effective [1]; however, there is limited long-term data on the effectiveness of switching to RTX half-dose from the real-world perspective.

Objectives: To compare the 3-year RTX regime retention between patients receiving retreatment with half-dose and full-dose of RTX, and assess factors associated with maintenance of $2 \times 500 \mathrm{mg}$, with a view to establish an optimal long-term retreatment strategy in RA.

Methods: An observational study was conducted on 755 consecutive RTXtreated RA patients in a single centre for over 20 years. Of these, 165/755 $(22 \%)$ received at least one cycle of $2 \times 500 \mathrm{mg}$. Long-term effectiveness was assessed using RTX retention rate. Drug survival of patients treated with $2 \times 500 \mathrm{mg}$ was compared with 200 RA patients receiving $2 \times 1000 \mathrm{mg}$ throughout the study, matched to the number of cycle when the $2 \times 500 \mathrm{mg}$ regime was initiated.

Results: Of the 165 patients who received $2 \times 500 \mathrm{mg}, 81.8 \%$ were female; mean age was 63.4 (26-91) years; mean disease duration 15.5 (2-53) years; 57 $(34.5 \%)$ were bDMARDs-naïve; 121 (74\%) were on concomitant DMARDs and mean DAS-28 was 3.9 (SD 1.29) at the half dose initiation. At 3 years, the retention rate was $38 \%$ for patients recieving $2 \times 500 \mathrm{mg}$ compared to $87 \%$ for those on 2x1000mg; $\mathrm{HR}$ for half dose discontinuation was 6.17 [95\% $\mathrm{Cl}(3.91-12.27)$; $\mathrm{p}<0.001$ ] (Figure 1A).

The main reasons for $2 \times 500 \mathrm{mg}$ discontinuation were poor EULAR response $(30 \%)$; moderate EULAR response $(30 \%)$; shorter duration of response compared to the full dose (22\%); and incomplete B-cell depletion (17\%). The majority of these patients (87.9\%) were switched back to $2 \times 1000 \mathrm{mg}, 5.1 \%$ received other bDMARDs and $7.1 \%$ did not receive further DMARDs.

In multivariable analysis, previous TNFi use was associated with 3-year maintenance of the $2 \times 500 \mathrm{mg}$ dose [OR 2.63 (1.12-6.10); $\mathrm{p}=0.024$ ]; while higher plasmablasts at $2 \times 500 \mathrm{mg}$ initiation was associated with shorter maintenance of this regime [OR $0.78(0.67-0.97) ; p=0.028]$.

There were no significant differences in 5-year RTX retention rates between patients receiving $2 \times 500 \mathrm{mg}$ and switched back to $2 \times 1000 \mathrm{mg}$ vs those receiving $2 \times 1000 \mathrm{mg}$ throughout the study (Log-Rank=0.186) (Figure 1B).

Conclusion: The use of RTX half-dose regimen was associated with poor retention at 3 years. Nevertheless, where loss of effectiveness occurs post$2 \times 500 \mathrm{mg}$ initiation, switching back to $2 \times 1000 \mathrm{mg}$ appears to be a pragmatic option. Patients with previous TNFi exposure and lower plasmablasts may be most suited to be commenced on the $2 \times 500 \mathrm{mg}$ dose for long-term disease control.

REFERENCES:

[1] Mariette X, et al. ARD 2014 
Figure 1A

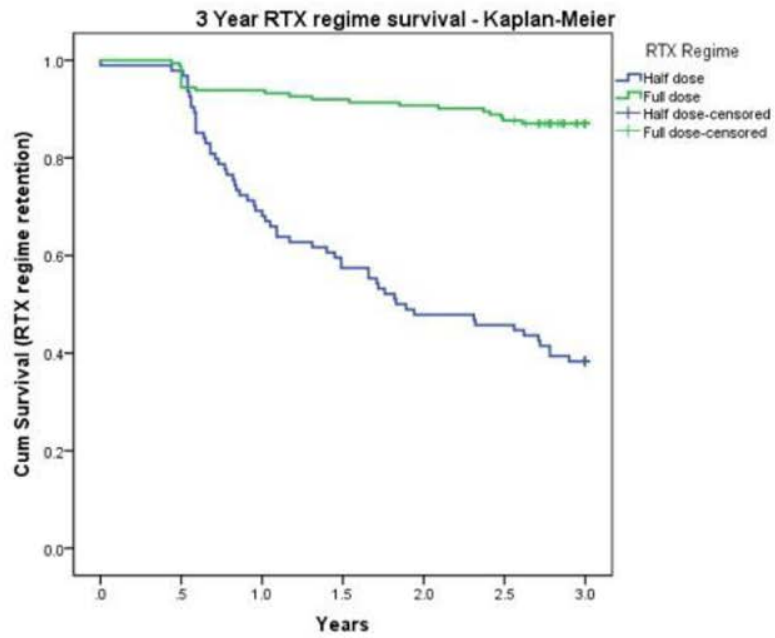

Figure 1B

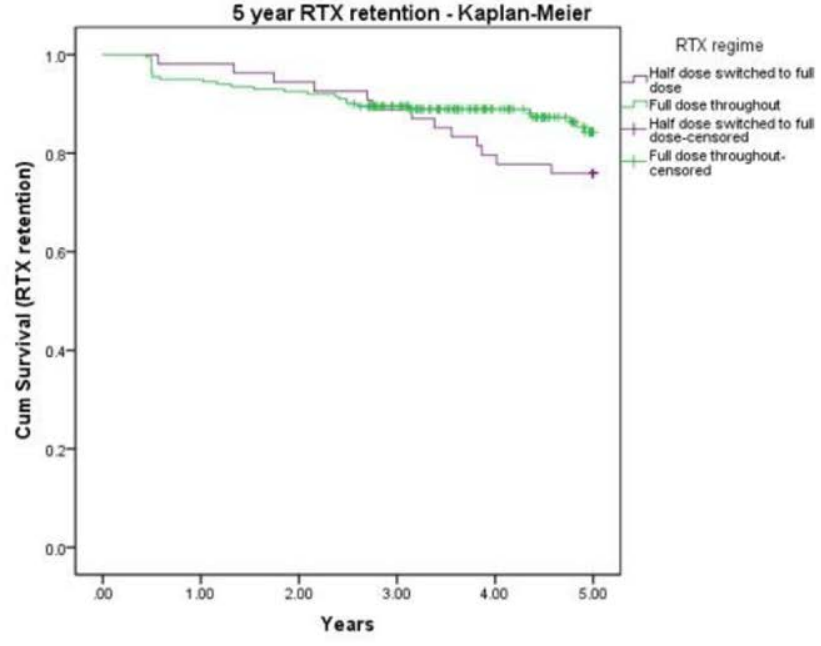

Acknowledgements: Leeds Cares

Disclosure of Interests: Leticia Garcia-Montoya: None declared, Md Yuzaiful Md Yusof: None declared, Gisela Eugénio: None declared, Jean Baptiste Candelier: None declared, Sudipto Das Speakers bureau: Dr Das has received honoraria from Roche, Edward Vital Speakers bureau: Dr Vital has received honoraria from Roche, Consultant of: Dr Vital has received honoraria a from Roche, Grant/ research support from: Dr Vital has received research grant support from Roche, Paul Emery Speakers bureau: Professor Emery has received honoraria from Roche, Consultant of: Professor Emery has received consultant fees from Roche, Grant/research support from: Professor Emery has received research grants paid to his employer from Roche.

DOI: 10.1136/annrheumdis-2021-eular.3691

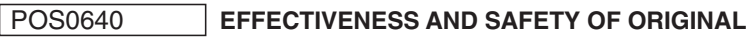 AND BIOSIMILAR ETANERCEPT IN BDMARD- NAÏVE PATIENTS IN A REAL-WORLD COHORT OF PORTUGAL}

A. S. Pinto ${ }^{1,2}$, M. Cunha ${ }^{3}$, F. Oliveira Pinheiro ${ }^{4}$, M. Bernardes ${ }^{4}$, H. Assunção ${ }^{5}$, J. Martinho ${ }^{6}$, M. C. Tenazinha ${ }^{6}$, A. M. Duarte Monteiro ${ }^{6}$, S. Silva ${ }^{7}$, F. R. Martins ${ }^{8}$, L. Silva ${ }^{9}$, M. Couto ${ }^{10}$, M. Faria ${ }^{11}$, F. Araújo ${ }^{12}$, T. Fontes ${ }^{13}$, D. Faria ${ }^{2}$, J. TavaresCosta ${ }^{2} .{ }^{1}$ Unidade Local de Saúde da Guarda, Rheumatology, Guarda, Portugal; ${ }^{2}$ Unidade Local de Saúde do Alto Minho, Rheumatology, Ponte de Lima, Portugal; ${ }^{3}$ Garcia de Orta Hospital, Rheumatology, Almada, Portugal; ${ }^{4}$ Centro Hospitalar Universitário de São João, Rheumatology, Porto, Portugal; ${ }^{5}$ Centro Hospitalar Universitário de Coimbra, Rheumatology, Coimbra, Portugal; ${ }^{6}$ Centro Hospitalar Universitário Lisboa Norte, Rheumatology, Lisboa, Portugal; ${ }^{7}$ Centro Hospitalar Baixo Vouga, Rheumatology, Aveiro, Portugal; ${ }^{8}$ Centro Hospitalar
Universitário do Algarve, Rheumatology, Faro, Portugal; ${ }^{9}$ Centro Hospitalar TrásOs-Montes e Alto Douro, Rheumatology, Vila Real, Portugal; ${ }^{10}$ Centro Hospitalar Tondela-Viseu, Rheumatology, Viseu, Portugal; ${ }^{11}$ Hospital Central do Funchal, Rheumatology, Funchal, Portugal; ${ }^{12}$ Hospital de Sant'Ana, Rheumatology, Parede, Portugal; ${ }^{13}$ Hospital do Divino Espírito Santo, Rheumatology, Ponta Delgada, Portugal

Background: The patent expiration of the original etanercept in Europe has facilitated the development of biosimilar products. Non-significant differences in efficacy and safety were noted in clinical trials which are not expected to influence clinical performance. Nonetheless, daily practice data should be gathered to support the claim for biosimilarity.

Objectives: To compare the effectiveness and safety of original and biosimilar etanercept, in biological-Disease Modifying Antirheumatic Drug (bDMARD)naïve patients.

Methods: A retrospective multicenter non-interventional study, using data collected prospectively from Reuma.pt (The Rheumatic Diseases Portuguese Register) was done, including patients with: age $\geq 18$ years old; diagnosis of Rheumatoid Arthritis (RA), Psoriatic Arthritis (PsA) or Spondyloarthritis (SpA) (axial or peripheral); active disease who initiated treatment with etanercept as first line of biological treatment after 2010. Kaplan-Meyer was used to calculate the persistence rate in treatment. Disease activity at baseline and follow-up data at $6,12,18$ and 24 months of treatment was compared using the chi-square for categorical variables and t-student or Mann-Whitney tests for continuous variables. Reasons for discontinuing therapy were summarized using descriptive statistics. Statistical significance was assumed for 2 -sided $p$-values $>0.05$.

Results: We included 1694 patients (413 on biosimilar and 1280 on original etanercept) 864 with RA, 335 with PsA and 494 with SpA. The population's baseline characteristics were not significantly different among both groups, except concomitant treatment in RA (higher use of conventional DMARDs in biosimilar group and higher use of NSAIDs in original group) and in SpA patients (higher use of corticosteroids in original group).

At baseline, a higher joint count was found in patients treated with original etanercept with a statistical difference for swollen $(p=0.03)$ and tender $(p=0.01)$ joints count (SJC and TJC, respectively) in RA and in TJC in SpA patients $(p=0.02)$. In RA patients, CDAI and SDAl were higher in patients who started original $(p=0.03$ $p=0.04$, respectively). Pain measured by visual analogic scale was higher in SpA patients treated with biosimilar $(p=0.03)$.

The 3-year PR was not significantly different between both treatment groups in RA, PsA and SpA (Figure1). In RA, PR in biosimilar was $72.6 \%$, with a median time-on-drug (TOD) of 28.3 months; for original etanercept PR was $63.6 \%$, with a median TOD of 27.4 months $(\mathrm{p}=0.566)$. In PsA patients, the PR for biosimilar was $70.6 \%$, with a median TOD of 27.6 months, and in original drug $67.0 \%$, with a median TOD of 28.1 months ( $p=0.743$ ). In SpA patients, the PR were $78.4 \%$ for biosimilar (median TOD of 27.4 months) and $71.5 \%$ for original treatment (median TOD of 28.0 months $(p=0.816))$.

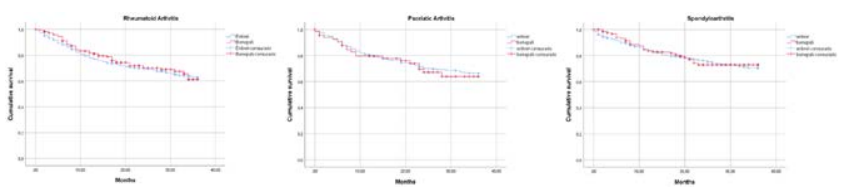

Figure 1. Drug survival in biosimilar and original etanercept in Rheumatoid Arthritis, Psoriatic Arthritis and Spondyloarthritis

In RA patients, we did not find differences between the two treatment groups for the proportion of patients in remission or low disease activity by CDAI $\leq 10$ $\mathrm{SDAl} \leq 11$ or DAS2 83.2 at $6,12,18$ and 24 months of treatment. For PsA, no differences were found in the same timelines for DAPSA $\leq 14$, DAS28<3.2, BAS $\mathrm{DAl}<4, \mathrm{ASDAS}<2.1$ or PsARC response. Also, in SpA patients, no differences were found in $B A S D A I<4, B A S F I<4, A S D A S<2.1$, ASDAS response and BASDAl response in all the timelines with the exception of BASDAI response at 18 months, which was achieved in fewer patients in biosimilar therapy $(p=0.02)$. Overall, $535(31.6 \%)$ patients stopped etanercept (428 patients on original and 107 patients on biosimilar). Discontinuations due to inefficacy were the most frequent, but there were no significant differences between both groups as for adverse events. Discontinuations due to "other reasons" were higher for the original group, both in RA ( $p=0.01)$ and in $\operatorname{SpA}(p=0.04)$.

Conclusion: Biosimilar and original used as first-line biological treatment showed similar effectiveness and safety in our long-term cohort of patients with RA, PsA, and SpA

Disclosure of Interests: None declared

DOI: 10.1136/annrheumdis-2021-eular.3706 